\title{
Can Design Documentaries Disrupt Design for Disability?
}

\author{
Seray B. Ibrahim \\ University College London, \\ London, UK \\ s.ibrahim@ucl.ac.uk
}

\author{
Asimina Vasalou \\ University College London, \\ London, UK \\ a.vasalou@ucl.ac.uk
}

\author{
Michael Clarke \\ University College London, \\ London, UK \\ m.clarke@ucl.ac.uk
}

\begin{abstract}
This paper shows how design documentaries can motivate new perspectives for design and disability. We critically consider the ways in which design documentaries can foreground children's lived experiences and priorities, in cases where it is not always possible to involve children early on in the design process. By presenting a design case for supporting communication that involves children with severe speech and physical impairments and their social peers, we discuss how this narrative method can evoke designer empathy and guide new interpretations. Our findings show that design documentaries can convey to designers rich and multifaceted accounts of children's communication experiences. Although this is found to be generative, we also identify a tension with a bodily impairment understanding of disability. Drawing on reflections from our case study, we propose new methodological implications for embedding design documentaries in the design process of technologies for disability.
\end{abstract}

\author{
Author Keywords \\ Disability; communication; design documentaries; children
}

\section{CSS Concepts}

- Human-centered computing Human computer

interaction (HCI); Interaction Design; Interaction design process and methods

\section{INTRODUCTION}

Children with severe speech and physical impairment (SSPIs) have limited opportunities for social interaction owing to the disabling interplay of their physical impairments and environmental barriers. In the 1960's, early electronic communication devices emerged as a way of supporting adults with acquired disorders, such as stroke, to replace or augment natural verbal speech through the option of electronic speech [29]. This same logic was then adopted for creating technologies for children with communication disabilities and it continues to underpin the design of contemporary augmentative and alternative (AAC) devices to date. AAC devices are technologies that are designed to offer opportunities for people to produce electronic speech derived through lexical or symbolic representations of language. More recently, interaction designers have been interested in contributing to the health and wellbeing of children with little or no functional speech by improving the design of existing AAC devices. However, with the growing volume of AAC technologies that focus on speech generation, yet the ongoing and widespread abandonment of these technologies by children and their families [2,14], it is both timely and important to consider whether there is scope for supporting children's communication experiences in new ways. Given the limited range of existing design perspectives, we are interested to pursue whether new communication technologies can be designed that go beyond the traditional functions that these technologies have taken in children's lives. Specifically we ask: can design documentaries assist in designing new communication technologies that transcend traditional views of AAC?

Our starting point is the desire to vividly convey to designers the lived communication experiences of children with SSPI so that children's priorities and motivations can generate new foci for design. To achieve this goal, we utilize design documentaries as a narrative design method for communicating rich, multidimensional accounts of people's lived experiences [25]. We demonstrate what a design documentary of children with SSPI can look like that foregrounds the ways that children have agency to communicate on their own terms. Next, we present a case study and evaluate how this design documentary is used by a design team who have had little exposure to designing for disability. We consider how generative this design method is in motivating new design perspectives on communication technology. Finally, we propose recommendations for how design documentaries can be used in designing for children with disabilities.

\section{RELATED WORK}

\section{Perspectives on designing for communication and disability}

Through a review of literature on interaction design and children over a period of 15 years, we identified four perspectives that have been prominent in technology design for children with diverse communication profiles and challenges including children with SSPI, learning difficulties and autism. We describe these perspectives with illustrative design examples. Our goal is to connect them to implicit and explicit theoretical perspectives on communication and disability with the view to map out the design space. 
Technology design for children's communication and disability has often followed the view that design can alleviate bodily impairment. This perspective has typically aspired to remediate bodily impairment by replacing, or augmenting, natural speech through the option of electronic speech. It originates from the medical model of disability that defines disability as residing in the individual [19]. It has been largely guided by an information transmission theory of communication that treats communication as a functional task. Technologies are thus designed to support the practical accomplishment of message transmission where a message is sent by one person and received by another via a mutually accessible channel, or signal (see [28] for a discussion).

Mankoff et al [19] argue that the impairment perspective has been highly prevalent in interaction design because it generates actionable solutions and clear ways of measuring change. For example, Madsen et al (2008) designed technology to help autistic adolescents interact with others 'in the moment' [18]. The designers observed that individuals with autism can have difficulties interpreting facial expression during their communication. They designed a tool to support adolescents with decoding facial expression information by capturing, analysing and conveying information about interlocutors' facial affect. In other work, Sampath et al (2012) and Lampe et al (2018) designed technologies to support non speaking individuals in message transmission using electronic speech [17, 26]. In the former case by making digitalised AAC cheaper and easier to edit and use on mainstream devices, and in the latter case by designing a portable text-to-speech solution that users could wear as a jacket.

An alternative perspective on design has taken a constructivist view recognizing that meaning is created in context. Accordingly, design can support situated, co-constructed communication. Avoiding a focus on deficit, disability and diagnosis, this perspective focuses instead on how interlocuters create meaning, whilst treating communication as a constant, dynamic process of meaning making $[6,16]$. In one example, Black et al utilised the distributed sources of everyday data that children could draw from when communicating to enrich and contextualise their conversations [7]. These designers used environmental sensors to collect data that was converted into text and speech and made available on children's existing AAC devices through natural language generation. The authors showed that environmental data sources could be utilised by interlocutors to foster conversational interaction. In other work, Barendregt et al [1] designed a forced collaborative interaction game to support the emergence of collaboration skills of children in special education settings. The game promoted a constant renegotiation of the children's shared goal, thus requiring children to adapt their communication based on their emergent choices in the game. These examples emphasise the role of interaction in allowing children to collaborate and/or jointly create meaning with other people as a dynamic process, supported by technology.

A third perspective on design prioritizes the view that design can support learning. Connected with a developmental perspective, designers have acknowledged that most children typically follow a broadly normative trajectory of development and that instruction through technology can support children in meeting developmental milestones. In one example of this, Borges et al [10] took a learning perspective on designing AAC for a child with cerebral palsy. The focus was on working with the school and therapy team to create a system that would support a child's language development based on the words and symbols that they were learning in school.

Lastly, other work has considered the role of self-expression in communication. This view has approached technology as a way to capture and communicate the complexities and nuances of children's daily experiences, in line with a critical disability view that promotes consideration of how the interplay between people and the environment creates disability. By capturing multiple life dimensions - including bodily impairment, social and cultural factors - designers seeking to facilitate selfexpression have attempted to bring visibility to different children's motivations, priorities, and experiences. A notable example is Durrant et al (2013) who explored how photographic practices might enhance the interpersonal communication of children with mixed abilities and their teachers.

\section{Design processes and tools}

The orchestration of design processes, and the role of the user have been the subject of much debate (e.g. [27, 30]). Historically, in user-centred design, the user, design researcher and designer took distinct roles. Design researchers acted as translators communicating outcomes from user research to the designer [27]. Increasingly, however, the value of a non-hierarchical and collaborative approach to design, where interdisciplinary teams jointly shape technology, has been recognised. Within the area of interaction design for children with disabilities and learning difficulties, [5] and [8] engaged in two separate systematic reviews of the existing literature. By mapping the terrain of design research for and with children with disabilities and learning difficulties, both reviews showed a growing volume of co-design. Yet, these reviews also showed that very few reported cases had considered how children's voices are heard and acted on early enough in the process to inform decision making.

Despite its importance, disabled children's participation in co-design has been difficult at times to attain. This has been particularly the case with children who have severe disabilities, such as children with SSPIs [8]. Frauenberger et al [11] reflect on the challenge of involving children with disabilities as co-designers throughout the design process. Chief concerns reported by Frauenberger and colleagues are the risk of overburdening children with the responsibility of maintaining a long-term design collaboration, and the lack of access to children. With the view to engender meaningful ways of participation, and to avoid 
tokenism, Benton and colleagues propose the importance of scaffolding neurodiverse children in personalised ways during the design process [4]. In our own research, we have highlighted the work involved in supporting children with SSPI's to participate in a design process - including the reflexive role of the researcher and the need to gain intimate knowledge of the child - toward gaining a deeper understanding of the child's voice [13].

The complexities of designing with users suggest the need to recognise and support a more diverse approach toward configuring how and when participation happens, echoing Vines et al [30] who captured the implicit and explicit ways in which user participation occurs in the design process. Framed in this view on participation, our paper explores the value of design documentaries - created to reflect the lived experiences of children with SSPI - in supporting designers to develop new perspectives on communication technology for this group of children. We engaged in a yearlong child-centred and participatory phase with children who have severe disabilities in order to understand their communication values, practices and relationships. Given the children's severe disabilities, and their protected time, we were not able to conduct co-design with them. Critical of the frequent use of proxies acting on behalf of this group of children (e.g. [5]), we wanted to support new ways of thinking about the design of their communication technology, that move beyond the deficit lens identified in the previous section, by building on the lived experiences of communication we documented in our research. To achieve this, we turned to design documentaries, a narrative method particularly apt in the early stages of design. Design documentaries support the designer to engage in open sense making and to develop new perspectives on design problems [32].

In the following sections, we first describe how we created a design documentary that would instil rich and multifaceted accounts of one child's communication experiences. We then explain how we used this with a team of designers tasked with creating a new communication technology concept for our child with SSPI. Finally, we reflect on how generative the tool is for motivating new and multiple perspectives in this design space. In particular, our goal was to understand if design documentaries can disrupt design for disability; and if so, how. In line with others, we define disruption as being able to interrupt and intervene in the traditional ways that technologies have been designed [31]. The next section describes the generative value of design documentaries, before describing how we created ours.

\section{A DESIGN DOCUMENTARY FOR A CHILD WITH SSPI}

\section{The value of design documentaries}

Narrative methods have been widely used in interaction design as a way of communicating the most important dimensions of user research to the designer. Narrative methods are designed to emphasize the importance of attending to experience as "an interplay of sensation, emotion, intellect and action situated in a particular place and time" [23, p.14]. Such methods provide the opportunity to portray well-rounded descriptions of people that allow the designer to engage deeply with characters and empathise with who is being designed for [22]. Though a range of narrative methods exist, most notably personas, our paper focuses on design documentaries introduced by Raijmakers et al and inspired by documentary film making [25].

Design documentaries are video stories in the form of a narrative, designed to convey multiple dimensions of people's lived experiences [25]. Given their qualities, we hoped design documentaries would be compelling in conveying rich accounts of children with SSPI, their communication experiences and details about idiosyncrasies that are part of their lives. Through documentary film approaches and techniques, design documentaries leave "the erratic, elusive fabric of the everyday intact" [ibid], which is useful in the early discovery stages of design. Thus, we approached design documentaries as a way to retain some level of ambiguity to encourage designers to imbue the video with their interpretation, while also making our perspective as film makers explicit.

In our role as film makers we wanted to portray an epistemological shift in interaction design that conceives communication as situated and co-constructed between child-partner [1]. In addition to this, informed by a social semiotic lens, we approached communication from a child's perspective; attending to the agentive ways that children create meaning on their own terms. For instance, rather than considering what children cannot do in line with their physical impairments, we instead wanted to convey what children can do with the resources that are available to them [15].

\section{Phase 1 - Research with a child with SSPI}

Over the course of a year, the first author visited a special school in a major UK city and engaged children with SPPIs in a mix of ethnographic and participatory research. The researcher's broad goal was to understand the salient features of communication and children's lived experiences. There are no typical cases of children with SSPIs. Therefore, rather than amalgamating the data of the five children who took part in the study, and to avoid the reduction of their unique experiences, our work focused on a single child to construct the design documentary: Grace, a primary school age girl with SSPI. Given the broad range of data that was generated with different children, we decided to focus on Grace who had the largest volume of data across the mixed methods we used. Both Grace and her parents provided informed consent, which was reaffirmed at the start of each research visit. One of the ways that we approached consent was by explaining the concept of information sharing. We used a 
posting box to represent the process of collecting and sharing ideas. Using this and pictorial support, we explained to Grace at the start of sessions that contributions would be shared with others interested in improving AAC.

Stakeholder involvement provided a starting point for planning research activities with children through face-to-face or telephone discussions with parents and teachers. In order to make sense of Grace's communication experiences, the following methods were used:

- Observation and transcription: providing a micro-lens on Grace (and other children's) classroom interactions with peers and teachers that were video-recorded;

- Fieldnotes: reflecting on events we were unable to video record, such as spontaneous games Grace participated in the playground, or interactions that occurred in corridors and between activities;

- Design workshops: that included making collages, storyboards and editing personas for the purposes of understanding the kinds of things that Grace (and other children) directly and indirectly expressed as important about themselves through how they responded to the methods.

\section{Phase 2 - Creating the design documentary}

A 5-min video was designed portraying Grace's communication. To design this, we drew from three sources: principles for designing narrative methods, theoretical perspectives that recognise communication as co-constructed and multi-modal, and data from the research documenting Grace's lived experiences.

The design documentary captured different elements of Grace's communication experiences. It suggested relations between specific events and a broader holistic view of what communication entailed for Grace on motivational, affective and practical levels. The video was made up of a series of short segments that together offered a macro view of Grace as a child with SSPI. We drew on the following sources: (1) short static or moving images of interactions with friends and school staff, (2) quotes about what others said about her (e.g. "You're brilliant! You need to show everyone that you're brilliant"), (3) verbal commentary, captions and interpretations by the researcher (e.g. "power struggles' / the caring friend", fig.1), (4) photos of craft-based artefacts that she and her friends had created in the design workshops and (5) researcher fieldnotes from a playtime interaction. Each source captured a different dimension of Grace's communication experiences.

The documentary represented a well-rounded account of Grace through detailed information that centred on her character rather than a plot [22]. This was done by communicating multiple dimensions about Grace and her everyday life. As the 5 minute video was intended to offer 'inspiration more than information' [25], it focused on conveying multiple dimensions from Grace's daily experiences that were considered as pertinent to her. Owing to the complexity of conveying a child-centred account for a child who uses minimal verbal speech, we decided to construct the video by drawing on multiple modes including narration, photographed events and artefacts, line drawing, video and written captions. The introduction to the video began with a narrated commentary by the researcher, introducing Grace's age, school setting, friends and family. The researcher narration explained that she had experienced changes to her brain development following oxygen deprivation at birth which now impacted on her bodily movements, accounting for her SSPI. The narration continued throughout the video, expressing Grace's personality and motivations for 'doing things the same as everyone else' as identified in the earlier fieldwork study. Still images and captions accompanied the verbal commentary to express how Grace appeared to feel about using certain communication modes (Fig. 1). This also included what she felt about her existing speech generating device and other modes, for example explaining: "Grace would rather not need to show everyone that she's brilliant" alongside the written caption: 'power struggles'.

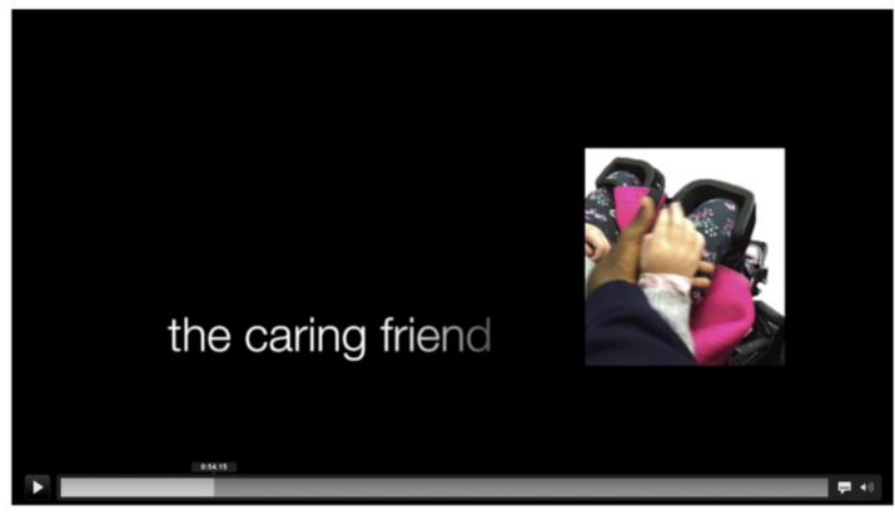

Figure 1. Video captions and images conveying friendship 


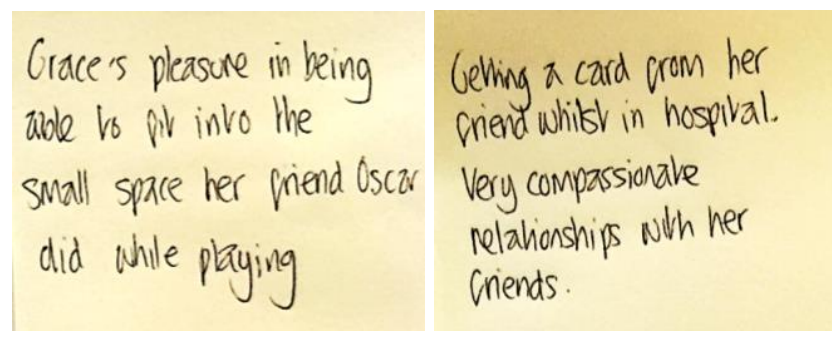

Figure 2. Example of identified critical moments

To minimise the chance of encouraging a deficit-oriented view of Grace, or making generalisations about specific conditions, clinical/medical descriptors and diagnoses were not used throughout. Thus, whilst detailed in places, there was very little information about the technical aspects of her existing speech generating device or her expertise relating to cognition, language levels or other normative features. With this in mind, we decided against including practical challenges that Grace encountered with daily tasks. Instead, the video documented what Grace could do, in line with a social semiotic perspective for communication that is socially shaped and reliant on culturally available resources for meaning making [6]. The different representational modes were important for evidencing the multiple ways in which Grace chose to communicate. The varied examples we drew from were intended to highlight the broad array of different ways she communicated with different people, as well as showing how she communicated with the resources available to her. For example, short video clips showed how she communicated through her whole body in class situations, whereas a narrated fieldnote entry from a playground event evidenced co-constructed communication that was shaped by available tools in the playground environment.

\section{CASE STUDY: EXAMINING THE GENERATIVE ROLE OF DESIGN DOCUMENTARIES FOR DISABILITY AND TECHNOLOGY}

\section{Context and participants}

Following others who have suggested that the worlds of design and disability could both inspire each other by embracing the cultures and sensibilities that they can each offer [24], we were interested in investigating how people from an arts school encountered disability, when utilizing the design documentary. We were motivated to involve designers who had less exposure to designing for disability, because in addition to contributing their design expertise, we felt that their minimal exposure to assistive technology would mean they would be more likely to approach activities without fixed perspectives or existing solutions in mind, that might otherwise inhibit new ways of engaging with communication and disability. The design study took place at a prominent arts college in the United Kingdom, widely considered as pioneering in its motivation to disrupt traditional ways of thinking about art and design.

Master's degree design students were contacted via their course tutor and invited to opt-in to the workshop. In total, eight participants consented to take part (seven students plus one tutor). The participants were arranged into two groups one of which (a group of three student participants) chose to pursue the design challenge beyond the workshop session. The outcome of this group's work is presented. Because we took a collaborative approach to design that recognized the design researcher and designers as jointly involved in shaping the outcome [27], the first author was also involved as a participant researcher.

\section{Design workshop}

We facilitated a three hour workshop that was structured around three phases for exploring perspectives on designing for communication and disability: 1. Understanding Grace's experiences, 2. Speculating and defining the problem space, and 3. Generating emergent design perspectives through iterative back-and-forth movements across these phases [23]. Following Raijmakers et al, (2006) the goal was to use the design documentary to develop more engaged intuitions and reflections about the child for whom communication technologies were being designed [25]. Workshop activities were organized in line with a design thinking approach involving activities that typically occur in the early stage of the design process. The activities comprised of a short presentation to the whole group, during which the participant researcher introduced the context of the design research and perspectives on disability. The design researcher then invited the team to watch the design documentary, and introduced the design challenge brief. A series of smaller group, structured activities then followed. The team were asked to identify and prioritize critical moments in the design documentary, followed by discussions to reflect on their prioritized decisions. The team proceeded to brainstorm how they might design solutions for the priorities they had identified in the design documentary, while being reminded to consider priorities that Grace and her social community might find valuable. At the end of the workshop, participants were invited to share their proposed solution, and encouraged to continue developing this beyond the workshop. Whilst the activities were structured to allow for moving through different design phases, we found that design students moved back and forth between these activities. As designer practices were not constrained to a linear process, the findings are described in terms of how designers engaged with the following three types of practices. 


\section{Understanding Grace's experiences}

Prior to introducing the design documentary, the participant researcher contextualized the design case by first presenting a summary of perspectives on designing for disability from prior design cases. In doing so, the intention was to invite participants to consider multiple starting points beyond the widely adopted view of designing for impairment or for traditional linguistic modes. As a group, participants then watched the design documentary together on a large screen to provide opportunities for sharing their initial thoughts. They were then provided with portable tablets so they could replay the video throughout the workshop. In the first phase, participants were invited to identify new possibilities for supporting Grace's communication. They were asked: 'Identify at least five critical moments in the video that are meaningful to the child. Describe what it is that characterizes the situation. You might focus on the child, the environment or something else.'

The design team used the design documentaries in a number of ways to create meaning. For instance, they identified critical incidents in the video that advanced the view of Grace being sociable and holding compassionate relationships with her friends. One designer suggested that Grace playing with her friend Oscar in the playground was key as she seemed the most engaged in the moment. Another team member identified Grace receiving a card from a friend who had been out of school in hospital for some time as another critical moment. She commented: "It's like they are very - sort of - extra compassionate kids, aren't they? I guess they've got a very grown up attitude to life." (see Fig. 2). These critical moments motivated their appreciation of Grace's social ties and her ability to access typical, everyday activities as well as expressing these social ties on an affective level.

While the design documentary was used to describe Grace, it was also used to generate questions. These questions would enable the design students to imagine who she is, and why she and her friends communicated in certain ways. For instance, whilst discussing an excerpt from a playtime interaction involving Grace and her friend Oscar, where Oscar had moved away without saying goodbye, one designer asked: "Is it because they're a child or because they have a disability?" By questioning the reasons why children acted in certain ways, the designers attempted to understand children's lives by considering different interpretations.

Like in the case of Raijmakers et al [25], designers were hungry for everyday details in seeking to understand how everyday, mundane events played out. They often came to the researcher to seek details and expressed "not knowing enough about her", suggesting a need to understand the intricacies of different aspects of Grace's daily life. For example, after identifying Grace receiving a card from her friend as an important moment, they were keen to understand how Grace and other children with SSPIs practically achieved specific actions, like communicating messages in a communication book or carrying out craft activities. Designers would direct questions to the participant researcher to understand the mechanics of how Grace constructed utterances with her communication book and AAC device. One designer commented:

"I wish I had seen technically how it works... how does the screen allow her to express an emotion... what does it sounds like too, a robotic voice or does it sound like an actual, normal - normal? - child's voice."

Furthermore, the design documentary also posed ambiguities and generated more questions. For instance, designers commented:

"I feel like I need to know more about her ... this is such a big task to take on ... it's hard to imagine for them, when you don't know them, and you've never been in their situation."

Comments such as these suggested that participants were keen to deeply engage in understanding what communication would be like for Grace and how they could make a positive impact on her life.

\section{Speculation and defining the problem space}

After identifying critical moments and having replayed the video a few times, participants were then invited to consider what role new technologies might take in Grace's life by responding to the instruction: "Using these identified moments, consider what it could mean for technology by forming statements: "technology could...". Consider are there similarities or differences in these moments?"

In Raijmakers et al [25], design documentaries allowed designers to concretely speculate about how individual people might be affected by their proposed ideas. By getting to know about Grace, designers could envisage how she might use a proposed technology, and consequently engage in loops of defining the problem space and testing out their speculations. During the workshop, the design documentary elicited three different types of speculation. These were: 1 . Speculating about how Grace might feel about her existing tech and new solutions; 2. Formulating 'what if' questions that considered how Grace's life might be affected by their proposed ideas, and; 3 . Speculating about contextual barriers that would impact on new ideas.

In the first case, designers used information in the design documentary to speculate about how Grace might feel, or what she would prefer, by making attributions. The design documentary was interpreted as suggesting that Grace's current AAC device was too large and not always helpful. This was evidenced through subtle narratives that foregrounded her more dominant 


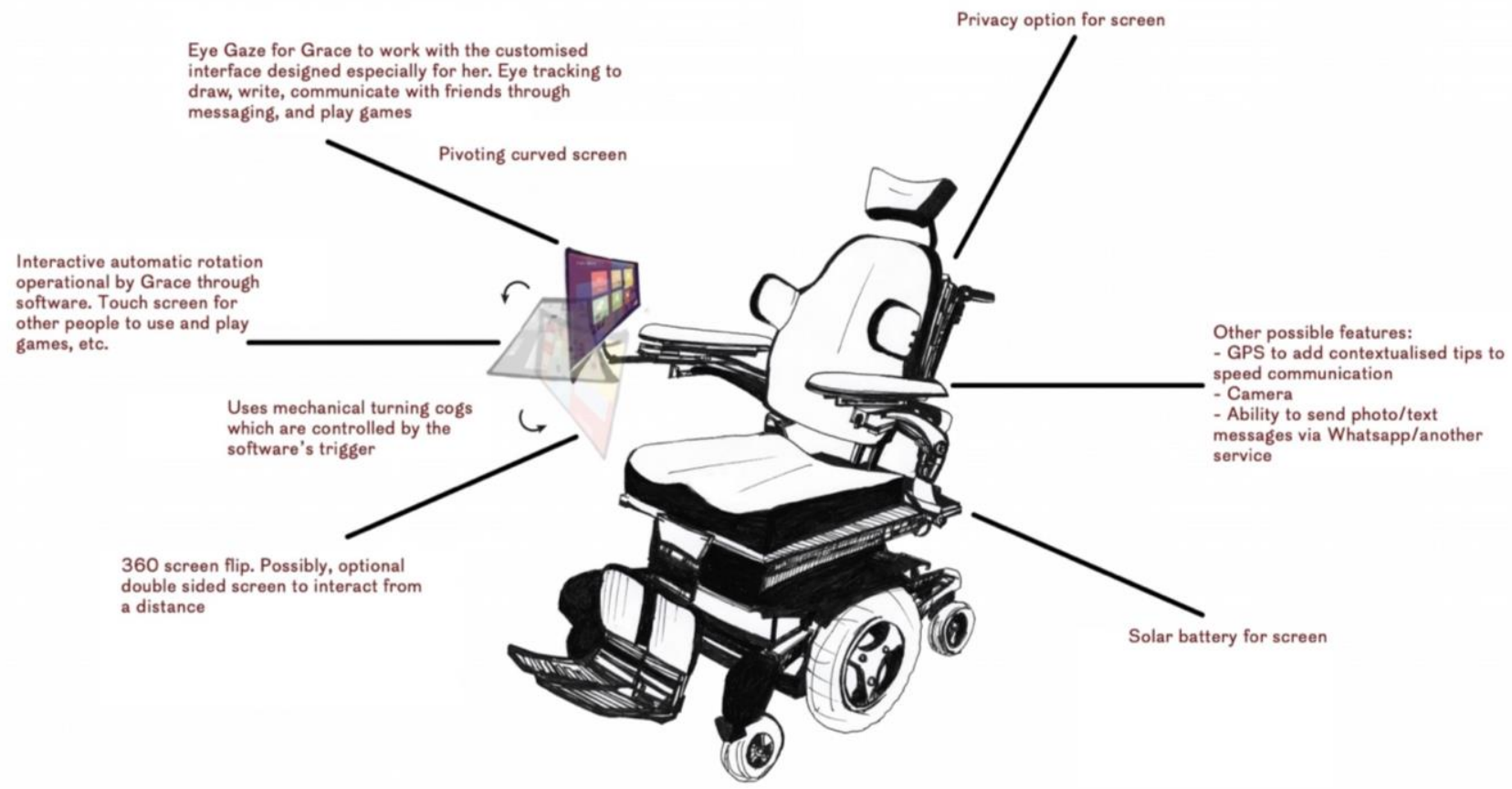

Figure 3. Design team's proposed idea: Whisper

communication methods (for example, eye pointing, facial expression and use of vocalization), as well as describing Grace as having "mixed feelings about her device". Taking these implicit suggestions as inspiration, the design team attributed these "mixed feelings" to a potentially invasive presence of technology that was imposed on her. The design team speculated about Grace's aesthetic tastes concerning technology by drawing on their own opinions about her technology being dated and cumbersome. They postulated that Grace would be likely to prefer something: "lighter... modular ... less of this big, black scary box she has" and "evidencing her personality a bit more, so it didn't feel so separate from her." By considering these alternatives, the team drew on their own interpretations and knowledge about the possibility of mainstream technologies to create a broader narrative about what Grace's technology preferences might look like.

During the workshop, 'what if' questions enabled designers to speculate about the impact of possible design solutions. Having established from the video some context about the kinds of activities Grace engaged in and the possibilities available to her, the team speculated about how things could be different for her with alternative possibilities. Some of these 'what if' questions included:

"what if money wasn't an issue?", "what if she could have one of those smart homes?" and "what if her equipment could evidence her personality a bit more?"

These questions led to discussion about the possible outcomes and broader impact that new technologies would have on her life. For example, one designer commented that by focusing on "evidencing her personality", new solutions could: "make [Grace] feel like she was being herself and wanting attention", as opposed to "hating being put on the spot." They also considered that new lightweight and

modular systems that Grace could independently regulate would "give her back control".

Designers also speculated about contextual barriers that they perceived would impact on Grace's communication experiences. Using the design documentary as a starting point for establishing basic information about her school and home environment for example, the team discussed the resource constraints of these settings. One designer asked whether all of the students in the school were children with disabilities. This helped to establish the scope of designing for certain communication experiences that included children of mixed abilities, therefore acknowledging the range of children's cognitive and physical impairments as potential barriers. The team also considered physical barriers that impacted on how Grace used her existing technologies. For example, when identifying key moments, the designers focused on Grace receiving a card from her friend. They used the design documentary to formulate questions that could only be probed by going to the participant researcher for clarification and detail. This was also discussed in Section 1 'Understanding Grace's experiences' where participants were keen to understand the intricacies of how everyday activities played out. 


\section{Emergent design perspectives and design concept}

Through engaging with Grace's experiences and speculating about her life, five perspectives were generated. These were:

- Speaking out in public and proving herself

- The importance of friendship

- Invasive technology and space issues

- Being included in shared play with common understandings

- Ethical, accountable design

Below we summarize these perspectives, highlighting where they link directly to issues expressed through the design documentary.

'Speaking out in public and proving herself' connected with a commentary from the video that expressed: "She hates being put on the spot...especially when there's as expectation that she'll use her eye gaze [device] to do so". The team proposed that Grace rejected her AAC device as it provided a means for adults to test her language capabilities. They also suggested Grace's existing device limited communication with other children on her own terms, through its restricted scope of modes and narrow volume range in regulating voice. This reflected a perceived imbalance of power dynamics between children and adults, exacerbated by Grace's AAC device.

'The importance of friendship' was prompted by the documentary that portrayed Grace as a caring friend with multiple peer connections. The team developed narratives about Grace's friendships that reflected this range and depth. They defined Grace's friendships in terms of who her friends were, what their social activities comprised of, and how they supported each other on an emotional level. Drawing on their own experiences of friendship, they noted that Grace had a wide range of friends compared to their own experiences at that age, and noted activities that strengthened Grace's social ties with other children.

'Invasive technology and space issues' were concerned with Grace's underuse of her existing AAC device, yet its obstructive physical presence. The participants suggested she should be able to regulate its presence. Drawing on points in the video that referenced to her house as being "too small" and "packed with all of her special equipment", the team discussed the impact that her existing technologies had on her home life.

Separately, 'being included in shared play with common understandings' referred to the importance of opportunities for creating bi-directional, co-constructed play. Utilizing the narrative describing a playground game of tag involving Grace, another child and the researcher, the team prioritized the importance of play as a right. They highlighted the importance of movement, speed and excitement in typical games like tag and considered play as a generational activity that helped to reinforce peer culture.

Finally, 'ethical, accountable design' was informed by their personal desire to produce solutions that were practically achievable within the scope of existing technologies, whilst designing with a motivation to 'do good'. For instance, one participant commented:

"I really wanna do things that help people... how we can change stuff". Also: "You don't want to disappoint them 'cause it's for kids"

These comments suggested that they did not want to let children down by making radical changes that they could not deliver on.

Utilizing different aspects from some of these perspectives, the team's final design concept, 'Whisper', allows Grace to communicate in 'quiet ways'. Quiet ways of communicating are subtle and multimodal, allowing for Grace and her peers to draw creatively on different means, according to the situation. This includes a range of private channels, including whispering, hiding games, passing notes to her friends in class and more. Focusing on voice, in particular, one of the designers described the design concept as a contextual way of regulating voice:

“...finding ways to allow Grace to control the volume she communicates at. Allowing her to speak more quietly or speak more loudly, depending on the situation".

Further, 'Whisper' also reflects a co-constructed view on communication by allowing for Grace to regulate the position of the device screen so that it can enable shared drawing on a flat surface or a two-way screen so that "peers can nonverbally interact with Grace in real time." Thus, broadly, the design concept is couched in the goal of providing Grace with control in how she regulates privacy in diverse activities related to communication, whilst also allowing for jointly constructed communication.

The design team's proposed idea is presented in figure 3. 'Whisper' closely aligns with reducing the need to "speak out in public and prove herself". It is motivated by cultural and social prompts that acknowledge the increasing presence of mobile technologies in young children's lives yet a need for these to move in and out of focus at different moments. Designing for 
advancing a peer culture that is separate from communicating with adults, 'Whisper' incorporates creative modes of communicating, such as drawing and playing games, whilst allowing for Grace to control the privacy of what others see. In order to achieve these intentions, and to offer solutions that are practically achievable, 'Whisper' draws on existing solutions for inspiration. For example, a pivoting screen mechanism allows for co-constructed communication as a shared tabletop surface, as well as improving battery life through solar power. The option of a curved and rotatable screen supports opportunities for sharing, whilst a blacked-out screen view supports privacy. Opportunities for situated meaning making are supported through location-based data that can generate contextualized 'tips' as well as speed up communication (figure 3). Finally, 'Whisper' incorporates an alternative writing function that is said to require minimal eye movement, for practicing free-hand writing with less physical effort.

\section{DISCUSSION}

Design documentaries are films created to inspire design thinking. On the one hand, they convey rich details about people's real world experiences, whilst on the other they present idiosyncrasies and ambiguity [25]. In this paper, we have shared our approach for constructing a design documentary and illustrated how it was used in a design case with participants who had little prior exposure to designing for disability, yet were tasked with supporting communication involving a girl with SSPI. In this section we reflect on our original goal to use design documentaries as a method to challenge existing design perspectives on technology for communication and children with SSPI. Based on these reflections, we suggest ways that design documentaries might be used in future design work where the voices of children with severe disabilities are of central concern, yet have been challenging to embed in the design process.

\section{Designing the documentary}

The construction of the design documentary was informed by principles for narrative design methods, theoretical perspectives on multimodal, co-constructed communication and data from earlier empirical research with children. The documentary aimed to engender polyvocality and multifaceted accounts of the communication experiences of a child called Grace. By piecing together a series of short narrative sequences involving different people, events, affective aspects and people's values, it aimed to open up the space for recognizing and making sense of critical moments in Grace's everyday life. Furthermore, rather than designing for communication from a developmental, linguistic or deficit-oriented perspective, the design documentary curated a holistic narrative that attended to Grace's agency in communicating on her own terms.

The documentary allowed us to express different layered perspectives on friendship, participation in social interaction and other foci, foregrounding these dimensions through the use of different media methods. These methods included videos, photos, drawings, written captions, audio narration and sound effects. To give one example, an image of a card that Maya (a classmate) had created for Grace communicated that Maya valued Grace as a caring friend, whilst a powerful photo of Maya and Grace holding hands, despite the physical effort to do so, captured that their friendship was bidirectional (fig. 1). Without the need for words, both of these instances clearly signalled some of the individualised ways that the girls enacted friendship and communicated on their own terms. Therefore, compared to static narrative methods, such as user personas, we were able to express a rich and dynamic portrayal of the child that itself went beyond language, using image and narrative sequence to convey the complexity of lived experience.

\section{How generative were design documentaries in designing for disability?}

For Dorst [9], generativity is concerned with applying multiple perspectives to help in understanding complex social problems. Accordingly, we intended for the design documentary to motivate new perspectives on designing for Grace's communication experiences, so that these perspectives could produce new outcomes that transcend the existing ways of designing for communication and disability. Prior work has shown that design documentaries offer a more 'dialectical' and 'interventional' approach by creating a narrative through the researcher's interpretive lens [12]. We found that our design documentary stimulated dialogue by generating speculations and questions that were useful for engaging with designer values concerning disability and childhood. This was important as the design documentary consequently became a key learning tool that highlighted team members' understandings about communication, disability, and Grace's experiences and providing opportunities for discussion about these areas. This approach builds on a growing body of design research that takes a critical and speculative angle on designing for disability (for example, [3, 20, 21]).

Using the information conveyed in the video as a starting point, the design team's discussions took into account the interplay between social, cultural and affective factors. For instance, considering the emotional impact of Grace's existing AAC device, the team proposed that Grace was frustrated by her technology being "imposed" on her for the practical accomplishment of talking, compared with her friends' uses of technology, which were "more varied, self-selecting and fun". As opposed to a functional perspective, the design team used the documentary to consider non task-based solutions that centered on designing for Grace's priorities as a young girl who valued friendships, play and doing things the same as everyone else. These designerly reflections showed that the design documentary disrupted existing modes of thinking and generated a layered and multifaceted perspective centring on the following themes: 
- $\quad$ Friendship - advancing its reciprocal and shared nature;

- $\quad$ Play - focusing on supporting common ground in ways that are both meaningful for Grace and her peers;

- Culture - that cultivates ways of advancing children's peer cultures that can be below the radar of adults;

- Social environments - minimising the invasiveness of assistive technologies, and;

- Accountable design - that acknowledges what is practically achievable.

These themes enriched and extended the perspectives that were identified in the literature review. In particular, the design team adopted a co-constructed lens on communication, and developed a narrative account that emphasized the social dynamics of children's friendships. Designers used the video to attend to Grace's subjective experiences and feelings, foregrounding a humanist agenda, which has been a key focus of narrative methods used in HCI [33]. For example, by engaging with what they treated as important for Grace, the design team's new perspectives addressed both her discontent in having to speak loudly in public via her AAC device, and apparent importance for her to be part of a peer culture through communication. This cultural dimension has traditionally not been previously discussed in designing for disability.

\section{Reflecting on the design team's outputs}

So far, we have argued that the design team's thinking was speculative and that the design documentary was facilitative of this speculation. However, the final design concept featured only part of this thinking. The design team prioritized "quiet ways of communicating" drawing on new perspectives of designing for peer culture and social relations. Therefore, although they had little experience in designing for disability, they contributed social, cultural and aesthetic sensibilities that have received little focus to date. Nonetheless, the proposed concept was also limited in its focus on existing technology. Specifically, part of the design team's recommendation utilized an existing screen-based AAC device that uses a grid layout for organizing symbol icons for words that is typically mounted on children's wheelchair. AAC devices emphasize bodily impairment by intervening at the level of the individual and providing the option of speech. Thus, in keeping with this solution, and its focus on language, the new ideas generated were layered on top of technology that has created tensions in emphasizing bodily impairment.

The design team often returned back to the notion of "doing good" and were driven by a sense of accountability toward the children. They desired to produce solutions that would evidence feasibility by being practically achievable. One designer explained: "you don't want to disappoint them 'cause it's for kids", which suggested that designers did not want to let children down by making radical changes they could not deliver on. This accountable stance and the team's desire to practically impact on children's lives created constraints that filtered out the speculative angle that had been generated through their reflective discussions, for instance, choosing not to pursue their idea of shared play that happens through the body. Therefore, whilst the final concept prioritized friendship and peer culture which were important dimensions for Grace, it relied on technologies that allow for augmenting communication through screen based message transmission, it did not transcend children's existing ways of communicating.

\section{Recommendations for embedding design documentaries in the design process}

In the current paper we detailed our approach to designing the documentary, unpacking both the practical work involved and the theoretical principles informing its design. We also reflected on the use of the design documentary as a method to motivate design teams to generate new perspectives on design, communication and disability. We identified an incongruence between how designers used the documentary as a dialogic tool and how they responded through their proposed solution. In this section, we reflect on our design process to problematize why this speculative angle was undermined, in order to set out our recommendations for using design documentaries in future design processes that support the design of technology in this space.

\section{Regulate how much additional information is provided}

The participant researcher who generated the design documentary acted as part of the design team. Designers prompted her for additional and specific technical information about Grace's existing AAC device and communication book system, knowing that the researcher had access to this information, having worked with Grace in the earlier phase. This introduced a tension between the emphasis we wanted to give in the documentary on Grace's lived experience and designers' desire to anchor this experience around Grace's existing communication technologies. Therefore, these extrinsic conversations may have impacted designerly interpretation by restricting the lens through which designers perceived Grace's communication. Future work should carefully consider strategies that allow for inhibiting oversharing of technical constraints.

Be consistent with the overall message that the various parts are conveying

The design documentary about Grace was intended to foreground her lived experience through the social and affective aspects of Grace's communication experiences. In hindsight, it also inadvertently generated a mixed message for the designers. In seeking to offer a brief introduction to Grace's physical impairment the introductory narrative began with: "there were complications which affected the oxygen flow to her brain" at birth. Despite our active decision to omit medicalised language, the inclusion of this information was out of kilter with the rest of the narrative. Arguably, the inclusion of this information may have spurred, or reinforced, designers to seek functional information about Grace's existing assistive technology and 
therapeutic tools. Design researchers who may want to use design documentaries as a method to move beyond a functional view technology should take care to create a consistent message throughout.

\section{Consider additional tools and strategies that can promote emergent design perspectives}

It is well established that designer values play an integral role in motivating design decisions e.g. [34]. In our case study, we found that designers' desire to 'do good' meant that they focused on existing solutions so that their proposed idea would be practically achievable. This happened despite them earlier drawing on a range of social and cultural sensibilities that could potentially have led to speculative ideas that transcend children's communication. In order to address this, we recommend that throughout the design process, explicitly engaging with designer values concerning disability with the goal to encourage transcendence. Through understanding their belief systems about disability, they can start to recognize how these shape these constraints.

\section{CONCLUSION}

This paper reported how design documentaries can motivate new perspectives for design and disability. We described our process for constructing and using a design documentary that was intended to foreground the lived communication experiences of a girl with SSPI. Our case study showed that design documentaries can convey to designers rich and multifaceted narratives about children's experiences that can motivate new dialogues for design. Our paper contributes to the field of interaction design and children in two ways. First it provides a methodological contribution by showing how design documentaries can be created and used in this design space, by amplifying child-centred perspectives that can be transformational in supporting opportunities for children's interactions and relationships. Second the design concept generated through the use of the documentary identifies new design opportunities for supporting communication involving children with SSPI that focus on friendship, play, cultural relations and social environments.

\section{ACKNOWLEDGMENTS}

This research was funded by the first author's ESRC doctoral studentship. We wish to thank Dr Laura Benton for her guidance and support in planning and carrying out the design workshop. We are also grateful to Professor Graham Pullin and Professor Carey Jewitt for their insightful and constructive feedback, informing the focus of this paper. We wish to thank the student design team and course tutor for their participation. Lastly, we are very grateful to the children, families and school who agreed to take part.

\section{SELECTION AND PARTICIPATION OF CHILDREN}

To inform the construction of the design documentary, in an earlier phase of the research, a critical case sampling approach was used to recruit one child with SSPI. This child attended a special school in the UK and was a user of an augmentative and alternative communication (AAC) technology. Informed consent was gained at the start of the research and reaffirmed at each research session. A mixed methods approach was taken, and thus the child participated in different forms of research including photo capturing, design workshops and observations.

\section{REFERENCES}

[1] Barendregt, W. et al. 2017. StringForce: A Forced Collaborative Interaction Game for Special Education. Proceedings of the 2017 Conference on Interaction Design and Children (New York, NY, USA, 2017), 713-716.

[2] Baxter, S. et al. 2012. Barriers and facilitators to the use of high-technology augmentative and alternative communication devices: a systematic review and qualitative synthesis: AAC barriers and facilitators review. International Journal of Language \& Communication Disorders. 47, 2 (Mar. 2012), 115-129. DOI:https://doi.org/10.1111/j.1460-6984.2011.00090.x.

[3] Bennett, C.L. and Rosner, D.K. 2019. The Promise of Empathy: Design, Disability, and Knowing the "Other." Proceedings of the 2019 CHI Conference on Human Factors in Computing Systems (New York, NY, USA, 2019), 298:1-298:13.

[4] Benton, L. et al. 2014. Diversity for design: a framework for involving neurodiverse children in the technology design process. (2014), 3747-3756.

[5] Benton, L. and Johnson, H. 2015. Widening participation in technology design: A review of the involvement of children with special educational needs and disabilities. International Journal of Child-Computer Interaction. 3-4, (Jan. 2015), 23-40. DOI:https://doi.org/10.1016/j.ijcci.2015.07.001.

[6] Bezemer, J. and Kress, G.R. 2016. Multimodality, learning and communication: a social semiotic frame. Routledge, Taylor \& Francis Group.

[7] Black, R. et al. 2012. Supporting Personal Narrative for Children with Complex Communication Needs. ACM Transactions on Computer-Human Interaction. 19, 2 (Jul. 2012), 1-35. DOI:https://doi.org/10.1145/2240156.2240163. 
[8] Börjesson, P. et al. 2015. Designing technology for and with developmentally diverse children: a systematic literature review. Proceedings of the 14th International Conference on Interaction Design and Children (2015), 79-88.

[9] Designer nights out: good urban planning can reduce drunken violence: 2016. http://theconversation.com/designernights-out-good-urban-planning-can-reduce-drunken-violence-52768. Accessed: 2019-07-07.

[10] de Faria Borges, L.C.L. et al. 2012. Customizing a Communication Device for a Child with Cerebral Palsy Using Participatory Design Practices: Contributions Towards the PD4CAT Method. Proceedings of the 11th Brazilian Symposium on Human Factors in Computing Systems (Porto Alegre, Brazil, Brazil, 2012), 57-66.

[11] Frauenberger, C. et al. 2011. Designing technology for children with special needs: bridging perspectives through participatory design. CoDesign. 7, 1 (Mar. 2011), 1-28. DOI:https://doi.org/10.1080/15710882.2011.587013.

[12] Green, D. et al. 2015. Interactive Design Documentary As A Method For Civic Engagement. Proceedings of the ACM International Conference on Interactive Experiences for TV and Online Video (New York, NY, USA, 2015), 161-166.

[13] Ibrahim, S.B. 2020. Communication and Non-Speaking Children with Physical Disabilities: Opportunities and Reflections from Design-Oriented Research. University College London.

[14] Johnson, J.M. et al. 2006. Perspectives of speech language pathologists regarding success versus abandonment of AAC. Augmentative and Alternative Communication. 22, 2 (Jun. 2006), 85-99. DOI:https://doi.org/10.1080/07434610500483588.

[15] Kress, G.R. 1997. Before writing: rethinking the paths to literacy. Routledge.

[16] Kress, G.R. 2010. Multimodality: a social semiotic approach to contemporary communication. Routledge.

[17] Lampe, R. et al. 2018. Mobile communication jacket for people with severe speech impairment. Disability and Rehabilitation: Assistive Technology. 13, 3 (Apr. 2018), 280-286. DOI:https://doi.org/10.1080/17483107.2017.1319427

[18] Madsen, M. et al. 2008. Technology for Just-in-time In-situ Learning of Facial Affect for Persons Diagnosed with an Autism Spectrum Disorder. Proceedings of the 10th International ACM SIGACCESS Conference on Computers and Accessibility (New York, NY, USA, 2008), 19-26.

[19] Mankoff, J. et al. 2010. Disability studies as a source of critical inquiry for the field of assistive technology. Proceedings of the 12th international ACM SIGACCESS conference on Computers and accessibility (2010), 3.

[20] Metatla, O. and Cullen, C. 2018. "Bursting the Assistance Bubble": Designing Inclusive Technology with Children with Mixed Visual Abilities. Proceedings of the 2018 CHI Conference on Human Factors in Computing Systems (Montreal QC, Canada, Apr. 2018), 1-14.

[21] Moser, I. 2006. Disability and the promises of technology: Technology, subjectivity and embodiment within an order of the normal. Information, Communication \& Society. 9, 3 (Jun. 2006), 373-395. DOI:https://doi.org/10.1080/13691180600751348.

[22] Nielsen, L. 2002. From User to Character: An Investigation into User-descriptions in Scenarios. Proceedings of the 4th Conference on Designing Interactive Systems: Processes, Practices, Methods, and Techniques (New York, NY, USA, 2002), 99-104.

[23] Plattner, H. et al. 2009. Design Thinking,. München (Mi-Verlag).

[24] Pullin, G. 2009. Design meets disability. MIT Press.

[25] Raijmakers, B. et al. 2006. Design Documentaries: Inspiring Design Research Through Documentary Film. Proceedings of the 6th Conference on Designing Interactive Systems (New York, NY, USA, 2006), 229-238.

[26] Sampath, H. et al. 2012. A Communication System on Smart Phones and Tablets for Non-verbal Children with Autism. Computers Helping People with Special Needs (Jul. 2012), 323-330. 
[27] Sanders, E.B.-N. and Stappers, P.J. 2008. Co-creation and the new landscapes of design. CoDesign. 4, 1 (Mar. 2008), 5-18. DOI:https://doi.org/10.1080/15710880701875068.

[28] Smith, M.M. and Murray, J. eds. 2016. The silent partner? language, interaction and aided communication. J\&R Press Ltd.

[29] Vanderheiden, G.C. 2002. A journey through early augmentative communication and computer access. Journal of Rehabilitation Research and Development. 39, 6 Suppl (Dec. 2002), 39-54.

[30] Vines, J. et al. 2013. Configuring Participation: On How We Involve People in Design. Proceedings of the SIGCHI Conference on Human Factors in Computing Systems (New York, NY, USA, 2013), 429-438.

[31] What is Disruptive Design? 2019. https://medium.com/disruptive-design/what-is-disruptive-design-5988e290ad88. Accessed: 2020-01-24.

[32] Wright, P. and McCarthy, J. 2008. Empathy and Experience in HCI. Proceedings of the SIGCHI Conference on Human Factors in Computing Systems (New York, NY, USA, 2008), 637-646.

[33] Wright, P. and McCarthy, J. 2010. Experience-centered design: designers, users, and communities in dialogue. Morgan \& Claypool Publ.

[34] Yarosh, S. et al. 2011. Examining values: an analysis of nine years of IDC research. (2011), 136-144. 